\title{
The Association between Genetic Polymorphisms and Simvastatin- Induced Myopathy: A Narrative Synthesis of Evidence
}

Authors

Xingang Lii, ${ }^{1}$, Shusen Sun ${ }^{3}$, Xiaowei Xu${ }^{4}$, Zhigang Zhao' ${ }^{1}$, Wei $\mathrm{Li}^{2}$

\section{Affiliations}

1 Department of Pharmacy, Beijing Tiantan Hospital, Capital Medical University, Beijing, People's Republic of China

2 Monogenic Disease Research Center for Neurological Disorders, Beijing Tiantan Hospital, Capital Medical University, Beijing, People's Republic of China

3 College of Pharmacy and Health Sciences, Western New England University, Springfield, Massachusetts, United States of America

4 Department of Cardiology, Beijing Tiantan Hospital, Capital Medical University, Beijing, China

Key words

simvastatin, genetic polymorphisms, myopathy, SLCO1B1, evidences

received $\quad 08.06 .2018$

accepted 06.08.2018

Bibliography

DOI https://doi.org/10.1055/a-0677-2944

Published online: 7.9.2018

Drug Res 2019; 69: 185-193

(c) Georg Thieme Verlag KG Stuttgart · New York

ISSN 2194-9379

\section{Correspondence}

Xiaowei Xu

Department of Cardiology

Beijing Tiantan Hospital

Tiantan Xili No. 6

Dongcheng District

100050 Beijing

People's Republic of China

Tel.: + 86/10/67098 353, Fax: + 86/10/67098353

E-mail: xuxiaoweittyy@sina.com

\section{Zhigang Zhao}

Department of Pharmacy

Beijing Tiantan Hospital

Tiantan Xili No. 6

Dongcheng District

100050 Beijing

People's Republic of China
Tel.: + 86/10/67098 036, Fax: + 86/10/67098 036

1022zzg@sina.com

Wei Li

Monogenic Disease Research Center for Neurological

Disorders

Beijing Tiantan Hospital

Tiantan Xili No. 6

Dongcheng District, 100050 Beijing

People's Republic of China

Tel.: + 86/10/67098694, Fax: 86/10/67098694,

Iwdoctors@sina.com

Supporting Information for this article is available online at

http://www.thieme-connect.de/products

\section{ABSTRACT}

Background and study aim Genetic polymorphisms may play a role in muscular injury associated with simvastatin, but results were inconclusive. This study aimed to summarize evidence from the literature investigating the effects of genetic polymorphism on simvastatin-induced myopathy.

Methods Studies regarding the association between genetic polymorphisms and simvastatin-induced myopathy were retrieved through electronic databases from February 1, 1990 to March 15, 2018. Two authors independently extracted data, including PMID, author, publication year, country, race, age, population characteristics, drugs, definition of case and control, gene, allele, SNP position, Hardy-Weinberg equilibrium, number of genotypes (case and control), minor allele frequency of cases and controls, association, study type and the Newcastle-Ottawa scale. Due to high heterogeneity in study design and outcome measurements among the included articles, a narrative synthesis of the evidence was conducted.

Results A total of 10 association studies were identified in this study, including SLCO1B1, ABCB1, GATM, HTR3B, HTR7, RYR2 and HLA-DRB1. The evidence linking myopathy to rs4149056 in SLCO1B1 is of high quality, and this association has been reproduced in randomized trials and clinical practice-based cohorts. As for other candidate genetic markers, the evidences are limited or controversial, and additional well-designed studies with larger sample sizes, are required to further elucidate this association. 
Conclusion SLCO1B1 genotype is a useful biomarker for predicting an increased risk of simvastatin-induced myopathy.

\section{Abbreviation}

SLCO1B1 Solute Carrier Organic Anion Transporter Family Member 1B1

ABCB1 ATP Binding Cassette Subfamily B Member 1

GATM Glycine Amidinotransferase

HTR3B 5-Hydroxytryptamine Receptor 3B

HTR7 5-Hydroxytryptamine Receptor 7

RYR2 Ryanodine Receptor 2

HLA-DRB1 Major Histocompatibility Complex, Class II, DR Beta 1

ABCG2 ATP Binding Cassette Subfamily G Member 2

\section{Introduction}

HMG-CoA reductase inhibitors, known as statins, are a class of lipidlowering medications. Until now, a number of statins are on the market, including atorvastatin, fluvastatin, lovastatin, pitavastatin, pravastatin, rosuvastatin and simvastatin. They have been found to reduce cardiovascular diseases and mortality in those who are at high risk of cardiovascular diseases [1]. The adverse effects of statins include muscle problems, an increased risk of diabetes mellitus, and abnormalities in liver enzyme tests [2], and the most important one being muscle pain [3]. Statin-related muscle problems include myalgias (pain without evidence of muscle degradation), myopathy (pain with evidence of muscle degradation), and rhabdomyolysis (severe muscle damage with acute kidney injury). The molecular mechanism of statin-induced myopathy is still unclear. Although the rate of muscle pain is low, the high prevalence of the clinical indication (hypercholesterolemia and cardiovascular disease) of its use creates a situation in which the absolute number of muscle pain is substantial.

Simvastatin is one of the most commonly prescribed statin formulation in the world. It has been reported that a single nucleotide polymorphism (SNP), rs4149056 T > C in SLCO1B1 increases the risk of muscle problem [4-9]. Additionally, published data also found that myalgia and rhabdomyolysis occur with greater frequency among carriers of the rs4363657C alleles in SLCO1B1 when taking simvastatin [5]. Except for SLCO1B1, other genetic markers were also investigated to explore the association, such as ABCB1 [10, 11], GATM [12, 13], HTR3B [14], HTR7 [14], RYR2 [15-17] and HLA-DRB1 * 04:06 [17]. Until now, the simvastatin-related muscle problem is unpredictable, and the evidence from literatures remained controversial.

We aimed to summarize the evidence from the literature about the association of genetic polymorphisms and simvastatin-induced myopathy, and to clarify which genetic markers can be useful for predicting an increased risk of simvastatin-induced myopathy.

\section{Methods}

\section{Search strategy}

The databases of PubMed, Web of Sciences, Embase, and Cochrane Library were searched. The search strategy of the following keywords or medical subject heading terms were used ("genetics" OR "pharmacogenetics" OR "polymorphism” OR "single nucleotide polymorphisms" OR “SNP” OR "variants” OR "variant”) AND ("myopathy" OR "myalgia” OR "myositis" OR "muscular diseases" OR “rhabdomyolysis" OR “creatine kinase” OR "CK”) AND ("statin" OR "simvastatin" OR "simvastatin acid" OR "hydroxymethylglutaryl-CoA reductase Inhibitors” OR “HMG-CoA reductase inhibitors”). The language was limited to English. We further searched the references cited in the included articles to identify other pertinent studies. Abstracts and unpublished studies were not considered. Because all analyses were based on previous published data, patient consent and ethical approval were not required.

\section{Study Selection}

Studies were included if they met the following criteria: 1) investigation of the association between genetic polymorphisms and risk of simvastatin-induced myopathy; 2 ) either a case-control or cohort study; 3 ) cases were defined according to the following criteria: a CK value $<3$-fold of the upper limit of normal (ULN) values with muscle symptoms (myalgia), or a CK value between $\geq 3$ and $<10$-fold of the ULN with muscle symptoms (myositis); or a CK value $\geq 10$-fold of the ULN with muscle symptoms (rhabdomyolysis); and 4) genetic tests were performed in all case and control patients. The following studies were excluded 1) patients without simvastatin treatment; 2) studies with patients < 3; 3) case report; and 4) patients with CK elevations that occurred in the setting of an acute coronary syndrome or myocardial infarction. The title and abstract of each study was evaluated by two reviewers (Xingang Li and Shusen Sun) independently. The final inclusion decision was made based on the consensus between the reviewers or consultation with a third reviewer (Wei Li).

\section{Data extraction and quality assessment}

Two reviewers (Xingang Li and Wei Li) independently extracted relevant information from each study. Any disagreements were resolved by discussion. A standard form was used for data collection, including PMID, author, publication year, country, race, age, population characteristics, drugs, definition of case and control, gene, allele, SNP position, Hardy-Weinberg equilibrium (HWE), number of genotypes (case and control), minor allele frequency of cases and controls, association, study type and Newcastle-Ottawa scale (NOS). NOS is used to assess methodological quality of cohort and case-control studies [18].

\section{Statistical analysis}

Only a few polymorphisms were reported by more than one study. Due to high heterogeneity in study design and outcome measurements among the included articles, an accurate and reliable metaanalysis could not be performed. Instead, we conducted a narrative synthesis of the evidence.

\section{Results}

\section{Study characteristics}

A total of 10 studies were identified in this study $[5,7,8,10-$ $14,16,17]$. The study selection process was shown in $\mathbf{F i g .} \mathbf{1}$. - Table 1 lists the main characteristics of included studies. Detailed information (PMID, author, publication year, country, race, age, population characteristics, drugs, case definition, control defini- 




Fig. 1 Flowchart of the study selection for inclusion in the systematic review of the association between genetic polymorphisms and simvastatin-induced myopathy.

tion, gene, alleles, SNP position, HWE, number of genotypes (case and control), minor allele frequency (MAF, case and control), association, study type and NOS) could be found in the Supplementary material 1. In most of the included studies, the genotype frequencies in the controls were consistent with the HWE. Results of the NOS showed that the methodological quality of the included studies were good (6-8 stars).

\section{Association between SLCO1B1 gene polymorphisms and simvastatin-induced myopathy}

SLCO1B1 gene encodes a liver-specific member of the organic anion transporter family. The encoded protein is a transmembrane receptor that mediates the sodium-independent uptake of endogenous compounds and drug compounds [19], including statins, from the blood into the hepatocytes [20]. SLCO1B1-dependent transport could well be important for the acid (active) form of simvas- 


\begin{tabular}{|c|c|c|c|c|c|c|c|c|c|c|c|c|c|c|c|c|c|c|}
\hline$\frac{\tilde{o}}{2}$ & $\infty$ & $\infty$ & $\infty$ & 0 & & $\infty$ & & $r$ & r & & $\wedge$ & & $\wedge$ & r & 6 & 6 & r & 0 \\
\hline 总 & 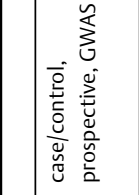 &  &  & 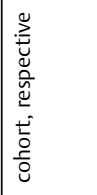 & & 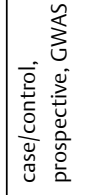 & & 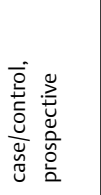 & 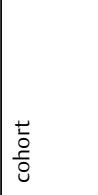 & & 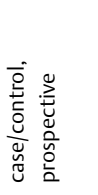 & & 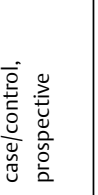 & \begin{tabular}{|l} 
蒿 \\
音
\end{tabular} & & 譬 & $\begin{array}{l}\text { 蒿 } \\
\text { 音 }\end{array}$ & 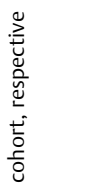 \\
\hline 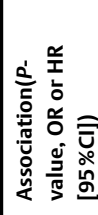 & 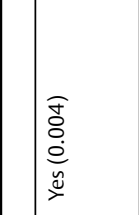 & $\begin{array}{l}\widehat{\tilde{O}} \\
\dot{\tilde{u}} \\
\check{\nu}\end{array}$ & 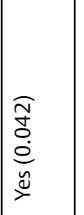 & 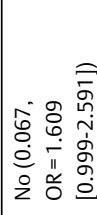 & & 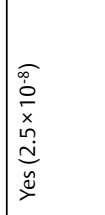 & & 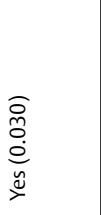 & 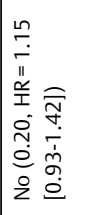 & & 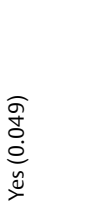 & & 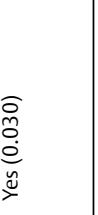 & 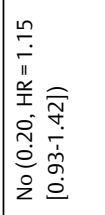 & &  &  & 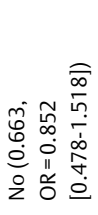 \\
\hline 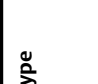 & ن & $\underline{z}$ & $\ddot{U}$ & $\frac{s}{z}$ & & $\frac{\tilde{z}}{z}$ & & $\stackrel{\widetilde{m}}{\stackrel{*}{*}}$ & $\frac{\pi}{z}$ & & $\stackrel{\bar{N}}{\ddot{F}}$ & & 嵃 & $\frac{\pi}{z}$ & $\Sigma$ & $\mathbb{z}$ & ن & $\Sigma$ \\
\hline & $\begin{array}{ll}\tilde{H} \\
\ddot{H}\end{array}$ & $\frac{\pi}{z}$ & $\begin{array}{l}\stackrel{\circ}{\circ} \\
\ddot{\ddot{U}}\end{array}$ & $\frac{x}{z}$ & & $\frac{s}{z}$ & & $\begin{array}{l}\mathscr{8} \\
\ddot{ت}\end{array}$ & $\frac{x}{z}$ & & 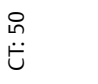 & & ن & $\Sigma$ & $\frac{\pi}{2}$ & $\mathbb{z}$ & $\begin{array}{l}0 \\
\ddot{z} \\
\end{array}$ & $\Sigma$ \\
\hline 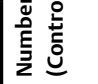 & 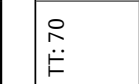 & $\frac{\pi}{z}$ & $\begin{array}{l}\tilde{N} \\
\ddot{F}\end{array}$ & $\frac{\pi}{z}$ & & ż & & $\bar{\sim}$ & $\frac{x}{z}$ & & $\stackrel{\infty}{\tilde{U}}$ & & 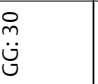 & $\underline{z}$ & $\frac{s}{z}$ & $\bar{z}$ & $\begin{array}{l}\tilde{z} \\
\ddot{z}\end{array}$ & $\underline{z}$ \\
\hline ڤ & $\begin{array}{l}\bar{N} \\
\ddot{U}\end{array}$ & $\frac{\pi}{z}$ & $\bar{u}$ & $\underline{z}$ & & $\underline{z}$ & & $\stackrel{\circ}{\ddot{F}}$ & $\underline{z}$ & & $\stackrel{\circ}{\ddot{F}}$ & & 萨 & $\frac{s}{z}$ & $\frac{\pi}{z}$ &  & $\begin{array}{l}n \\
\ddot{j} \\
ن\end{array}$ & $\underline{z}$ \\
\hline 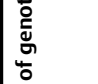 & $\begin{array}{l}\stackrel{n}{m} \\
\stackrel{t}{U}\end{array}$ & $\underline{z}$ & $\begin{array}{l}0 \\
\ddot{U} \\
\ddot{U}\end{array}$ & $\frac{\pi}{z}$ & & $\underline{z}$ & & 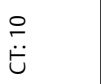 & $\underline{z}$ & & 음 & & 点 & $\frac{\pi}{z}$ & $\bar{z}$ & $\frac{\mathbb{s}}{z}$ & $\begin{array}{l}n \\
\\
\ddot{z}\end{array}$ & $\underline{z}$ \\
\hline 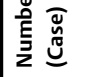 & $\begin{array}{l}\stackrel{2}{2} \\
\stackrel{F}{F}\end{array}$ & $\frac{\pi}{z}$ & $\stackrel{n}{\mathscr{H}}$ & $\frac{s}{z}$ & & $\underline{z}$ & & نُ & $\frac{\pi}{z}$ & & نُ & & تُ & $\underline{z}$ & $\bar{z}$ & $\bar{z}$ & 䒰 & $\underline{z}$ \\
\hline 岁 & $\stackrel{\check{\nu}}{\check{\nu}}$ & $\stackrel{\mathscr{\nu}}{\partial}$ & $\stackrel{\check{\nu}}{\check{\nu}}$ & $\underline{z}$ & & $\stackrel{\varpi}{\nu}$ & & $\stackrel{\varpi}{\check{\nu}}$ & $\stackrel{\breve{\nu}}{\nu}$ & & $\stackrel{\check{\nu}}{\supset}$ & & $\stackrel{\check{\nu}}{\nu}$ & $\stackrel{\check{y}}{\check{0}}$ & & 气ूّ & $\stackrel{\breve{u}}{\check{0}}$ & $\frac{\pi}{z}$ \\
\hline 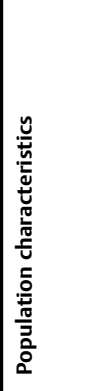 &  & 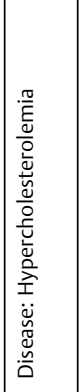 & 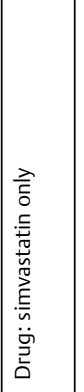 & 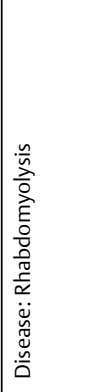 & & 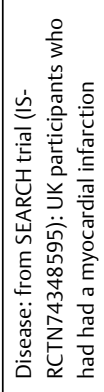 & &  & 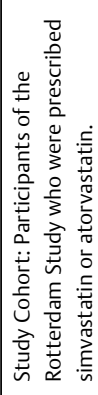 & & 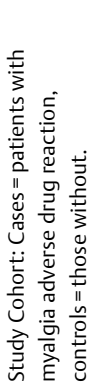 & &  & 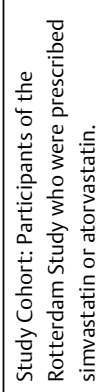 & &  & 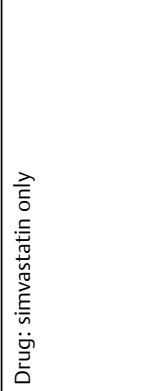 & 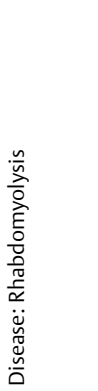 \\
\hline 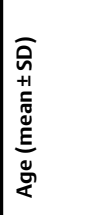 & $\frac{\pi}{z}$ &  & 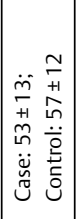 & 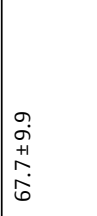 & & $\frac{x}{z}$ & & 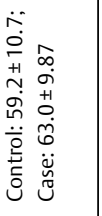 & 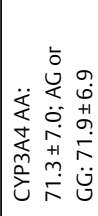 & &  & & 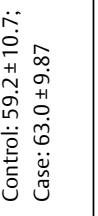 & 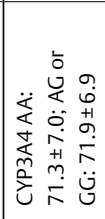 & $\frac{\pi}{z}$ & $\bar{z}$ & 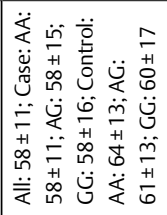 & 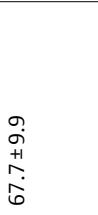 \\
\hline  & 茕 & 蒙 & 蒙 & $\frac{\frac{5}{6}}{\frac{60}{2}}$ & & 蒙 & & 茕 & 竞 & & 蒙 & & 華 & 站 & 范 & 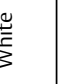 & 蒙 & $\frac{\frac{5}{5}}{\frac{0}{4}}$ \\
\hline 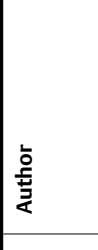 & 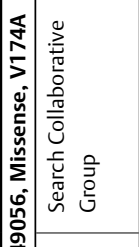 & \begin{tabular}{|l}
0 \\
0 \\
0 \\
0 \\
$\vdots$ \\
$>$
\end{tabular} & 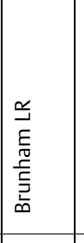 & 总 & 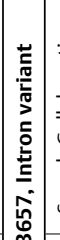 &  & 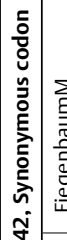 & 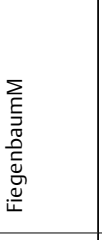 & 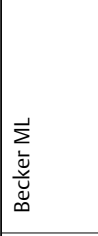 & 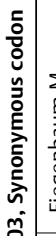 & 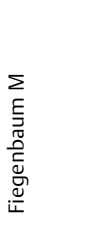 & 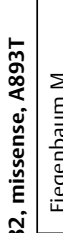 & 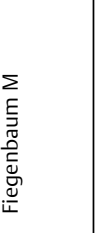 &  & 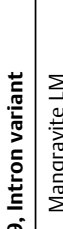 & 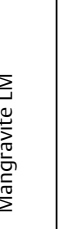 & 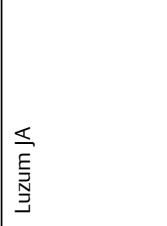 & 莣 \\
\hline 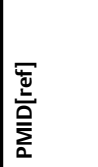 & 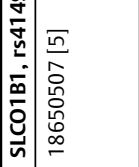 &  & 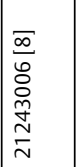 &  & 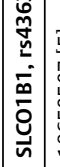 & 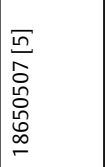 & 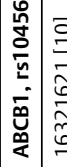 & 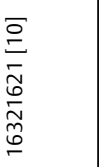 &  & 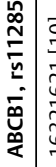 & 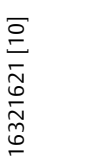 & 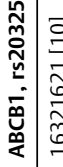 &  & 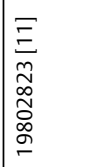 & 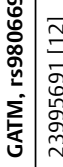 & 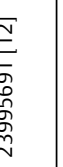 & $\begin{array}{l}\bar{m} \\
\underline{\Xi} \\
\bar{n} \\
\tilde{\tilde{D}} \\
\infty \\
\stackrel{N}{N}\end{array}$ & 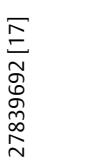 \\
\hline
\end{tabular}




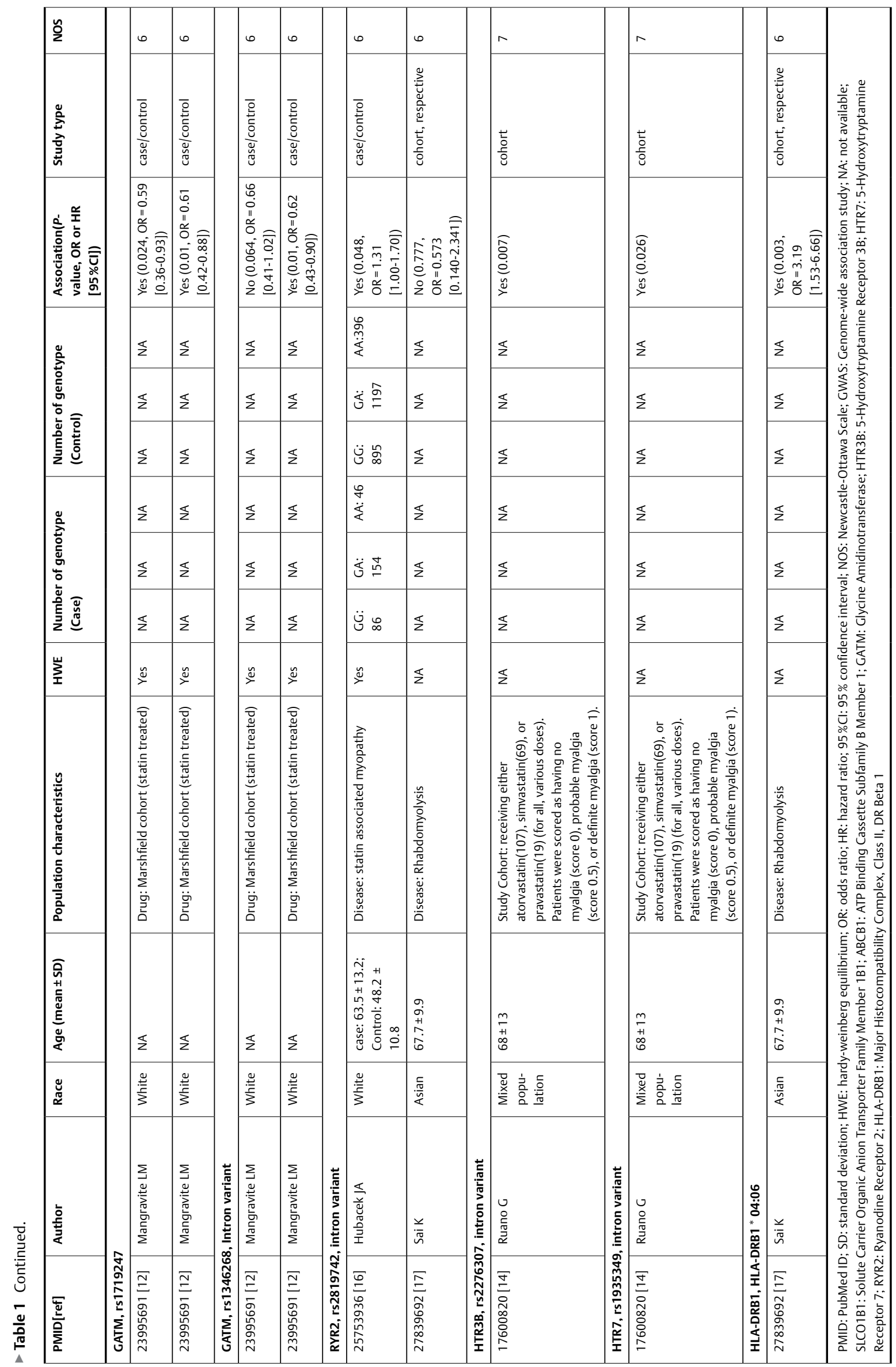


tatin. Polymorphisms in this gene encoding-protein are associated with an impaired transporter function.

A total of 4 studies reported the association of SLCO1B1 gene polymorphisms and simvastatin-induced myopathy, including rs4149056 and rs4363657. The Study of the Effectiveness of Additional Reductions in Cholesterol and Homocysteine (SEARCH) Collaborative Group conducted a case-control study of simvastatininduced myopathy using archived DNA from a randomized trial including more than 12,000 subjects who had received simvastatin post-myocardial infarction. This genome-wide study first identified common variants (rs4149056 and rs4363657) in SLCO1B1 that were strongly associated with an increased risk of simvastatin-induced myopathy [5]. The noncoding rs4363657 SNP was in nearly complete linkage disequilibrium with the nonsynonymous rs4149056 SNP $\left(r^{2}=0.97\right)$, which was linked to statin metabolism. The following study reported that the carriers of the SLCO1B1 ${ }^{*} 5$ allele (rs4149056) were at a two-fold relative risk of mild statin induced side effects-the majority of which had normal CK levels [7]. This association was also replicated in a cohort of patients with severe statin-associated myopathy. The rs4149056 variant in SLCO1B1 was not significantly associated with myopathy in that group as a whole. The SLCO1B1 rs4149056 genotype was not significantly associated with myopathy in patients who received atorvastatin [8]. However, another pharmacogenetic research in the Asian population found that no significant association of SLCO1B1 variants with statin-related myopathy were observed in the Japanese patients [17]. Since simvastatin was not the major drug used by their statin-related myopathy cohort (5/52) and the commonly used dosages of statins were lower in Japan, usually half, than those administered in the USA, these might have influenced the results. Considering the significant impact of this single coding single nucleotide polymorphism, rs4149056T > C, in SLCO1B1 on the increased risk of muscle toxicity, the Clinical Pharmacogenetics Implementation Consortium (CPIC) summarized evidence from the literature supporting this association and provide therapeutic recommendations for simvastatin based on SLCO1B1 genotype [6].

\section{Association between $A B C B 1$ gene polymorphisms and simvastatin-induced myopathy}

The membrane-associated protein P-glycoprotein (P-gp) encoded by $A B C B 1$ gene is a member of the superfamily of ATP-binding cassette (ABC) transporters, and it is an ATP-dependent drug efflux pump for xenobiotic compounds with broad substrate specificity [21]. ABCB1 is expressed in the wall of intestine and in the liver, and transports simvastatin out of the intestine wall into the lumen, and out of the hepatocytes into the bile. After transportation into the lumen or into the bile, the simvastatin is still available for re-absorption and uptake in the circulation.

Two studies investigated the interactions between common polymorphisms in ABCB1 genes and the safety of simvastatin. The 1236 T, 2677non-G, and 3435 T alleles were less frequent in adverse drug reaction (ADR) cases than in the non-ADR group ( $P$-value $<0.05$ for all SNP). Haplotype analyses also demonstrated a reduction of the T-non-G-T haplotype frequency (20\%) in patients in whom myalgia developed during simvastatin treatment, as compared with the non-ADR group (41.4\%) (P-value $=0.03)$ [10]. This result was not supported by the following study, and the ABCB1
C1236T, G2677A/T, and C3435T polymorphisms did not affect the risk [11]. Therefore, additional studies will be needed to confirm the association.

\section{Association between GATM gene polymorphisms and simvastatin-induced myopathy}

GATM encodes glycine amidinotransferase, a rate-limiting enzyme in creatine synthesis [22]. Creatine plays a vital role in the energy metabolism in muscle tissues. GATM knockdown in hepatocytederived cell lines attenuated transcriptional response to sterol depletion, demonstrating that GATM may act as a functional link between statin-mediated cholesterol lowering and susceptibility to statin-induced myopathy [12].

The locus rs9806699 is an expression quantitative trait locus (eQTLs) for the gene GATM. The association of the GATM eQTL locus with statin-induced myopathy was investigated in a populationbased cohort comprised of 72 cases of myopathy and 220 matched controls (Marshfield cohort)[23]. In that cohort, the minor allele at the GATM eQTL locus was associated with a reduced incidence of statin-induced myopathy (odds ratio, OR, $=0.61,95 \%$ Confidence Interval $(\mathrm{Cl})=0.39-0.95, \mathrm{P}$-value $=0.032)[12]$. Three SNPs rs9806699, rs1719247 and rs1346268 were in linkage disequilibrium $\left(r^{2}=0.70-0.80\right)$. This association was also observed in a cohort consisting of 100 cases of myopathy identified within the SEARCH [5]. But GATM gene polymorphism (rs9806699) associated with the risk for statin-induced myopathy was not replicated in the casecontrol analysis of 715 dyslipidemia individuals [13]. No significant association of GATM variants (rs9806699) with statin-induced myopathy were observed in the Japanese patients [17].

\section{Association between RYR2 gene polymorphisms and statin-induced myopathy}

RYR2 gene encodes a ryanodine receptor, and the encoded protein is one of the components of a calcium channel. Calcium channel that mediates the release of $\mathrm{Ca}^{2+}$ from the sarcoplasmic reticulum into the cytoplasm [24], and thereby plays a key role in triggering muscle contraction. Two studies reported the association between PYR2 rs2819742 (G > A) and statin-induced myopathy. Hubacek et al. reported a study in Czech 288 adult patients with statin-induced myopathy (on simvastatin or atorvastatin with doses of 10 or $20 \mathrm{mg}$ per day) and compared the allele frequencies of the SNP between this group and a group of 2,492 healthy adult controls not on statins [16]. Allele $A$ is associated with an increased likelihood of muscular diseases and myalgia unspecified when exposed to atorvastatin or simvastatin in patients as compared to allele $\mathrm{G}$ ( $\mathrm{P}$ value $=0.048$ ). However, this association was not replicated in the Asian patients, and no significant association of RYR2 variants with statin-related myopathy was observed in Japanese patients ( $\mathrm{P}$-value $=0.777)$ [17].

\section{Association between HTR3B and HTR7 gene polymorphisms and statin-induced myopathy}

The product of HTR3B belongs to the ligand-gated ion channel receptor superfamily GO annotations related to this gene including ion channel activity and G-protein coupled serotonin receptor activity [25]. The serotonin receptor encoded by HTR7 belongs to the superfamily of G protein-coupled receptors [26]. Until now, only 
one study investigated the association between HTR3B and HTR7 gene polymorphisms and statin-induced myopathy [14]. Patients receiving either atorvastatin $(n=107)$, simvastatin $(n=69)$, or pravastatin ( $n=19)$ (for all, various doses) were scored as having no myalgia (score 0 ), probable myalgia (score 0.5 ), or definite myalgia (score 1). SNPs in the HTR3B and HTR7 genes, rs2276307 and rs1935349, respectively, were significantly associated with the myalgia score. For rs2276307 ( $>$ $>G$ ), the $G$ allele was associated with a higher myalgia score, with allele frequencies the highest in those with definite myalgia and the lowest in those with no reported myalgia $(P$-value $=0.007)$. For rs1935349 $(C>T)$, the T allele was associated with a higher myalgia score $(P$-value $=0.026)$. Individual differences in pain perception and nociception related to HTR3B and HTR7 gene variants may affect the development of myalgia in statin-treated patients.

\section{Association of HLA-DRB1 * 04:06 and statin-induced myopathy}

HLA-DRB1 plays a central role in the immune system by presenting peptides derived from extracellular proteins [27]. Only one study reported the association of HLA-B1 * 04:06 and statin-induced myopathy [17]. A significantly (OR [95\% Cl] = 3.19 [1.536.66], P-value $=0.003$ ) higher carrier frequency of HLA$\mathrm{DRB} 1{ }^{*}$ 04:06 was detected in statin-related myopathy patients $[0.173(9 / 52)]$ compared with that in the controls $[0.062$ (177/2878)], even after adjustment for multiple comparisons (corrected $\mathrm{P}$-value $=0.045)$. HLA-DRB1 ${ }^{*} 04: 06$ play a role in an increased risk of muscular diseases when treated with atorvastatin, fluvastatin, pitavastatin, pravastatin, rosuvastatin or simvastatin as compared to HLA-DRB 1 * 01:01. This study demonstrated for the first time a possible association of HLA-DRB1 ${ }^{*}$ 04:06 with statinrelated myopathy in the Asian population. However, simvastatin was not the major drug used by their statin-related myopathy cohorts. Although a validation study is clearly needed, these findings may provide further insight into understanding the importance of immune-mediated mechanisms of statin-related myopathy, and into investigating appropriate ethnicity-related genetic markers.

\section{Discussion}

The SLCO1B1 gene spans fifteen exons and 190 common variants with a minor allele frequency $>5 \%$ have been identified. Of these, two common nonsynonymous SLCO1B1 variant have been well characterized: rs2306283 (SLCO1B1: 492 A > G on NM_006446.4, N130D) and rs4149056 (SLCO1B1: 625 T > C on NM_006446.4, V174A). The common c.521 T>C variant, rs4149056, produces a p.V174A substitution. The minor $C$ allele at this locus has been associated with a decreased transport function in vitro[28, 29] and a decreased clearance for a number of drugs in vivo4, 30]. The FDA recommends against $80 \mathrm{mg}$ daily simvastatin dosage. In patients with the C allele at SLCO1B1 rs4149056, there are modest increases in myopathy risk even at lower simvastatin doses ( $40 \mathrm{mg}$ daily); if optimal efficacy is not achieved with a lower dose, alternate agents should be considered. The 2014 update of CPIC guideline regarding SLCO1B1 and simvastatin-induced myopathy, has been published in Clinical Pharmacology and Therapeutics [6]. In case group, the MAF was much lower in Asian population (0.212) compared to White pop- ulation (0.330-0.453) (Supplemental material 1). For simvastatin, the evidence linking myopathy to rs4149056 in SLCO1B1 is of high quality, and this association has been reproduced in randomized trials and clinical practice-based cohorts. Conversely, the association of rs4149056 with myopathy has been less compelling for other statins [31]. The impact of rs2306283 polymorphism on the efficacy of statins has been reported, such as pravastatin [31,32], atorvastatin [33-36], and pitavastatin [37]. However, no report regarding the correlation of rs 2306283 polymorphism and simvastatin-induced adverse reaction was published until now. The SNP of rs4363657 is located in the intron (g.T89595C, intron 11) of SLCO1B1. This noncoding rs4363657 SNP was in nearly complete linkage disequilibrium with the nonsynonymous rs4149056 SNP $\left(r^{2}=0.97\right)$, which has been linked to statin metabolism [5].

Mutations in ABCB1 may play a role in statin-induced myopathy. The three most common SNPs in the protein coding region are rs1128503 (1236 T > C, Gly412Gly), rs2032582 (2677 T > G/A, Ser893Ala/Thr), and rs 1045642 (3435 T >C, Ile1145lle) [38]. These three SNPs have been the focus of many pharmacokinetic and disease association studies with controversial results [39]. Kajinami $\mathrm{K}$ et al. found the polymorphisms in $A B C B 1$ gene influence the response to atorvastatin treatment [40]. The association of $A B C B 1$ gene polymorphisms, and efficacy and safety of simvastatin and atorvastatin were identified later $[10,11]$.

It was first reported that a variant within the RYR2 gene plays an important role in life-threatening rhabdomyolysis after cerivastatin (withdrawn from the market due to its serious side effect) administration [15]. This association was identified in Caucasian patients on low doses of simvastatin or atorvastatin.

HTR3B receptor causes fast, depolarizing responses in neurons after activation. Among HTR7's related pathways are calcium signaling pathway and $\mathrm{G}$ alpha(s) signaling events. Serotonin 5-HT7 receptors are located primarily in the thalamus, hypothalamus and hippocampus. The function of these receptors includes the regulation of circadian rhythms, thermoregulation, learning and memory, and smooth muscle relaxation. The results indicate that gene polymorphisms producing individual differences in pain perception, and this may have an important role in the statin-induced muscle pain.

Hundreds of DRB1 alleles have been described. A significant association was detected for HLA-DRB1 * 04:06 with statin-related myopathy in the Japanese patients. This result provided an additional insight regarding the importance of immune-mediated mechanisms of statin-related myopathy. However, the allele frequency of HLA-DRB1 * 04:06 is relatively higher in Asians (1-5\%) compared with that in Caucasians (0-0.3\%) and African Americans (about $0.06 \%$ ) (http://www.allelefrequencies.net/default.asp), this marker may only be useful for Asian populations.

In addition, different effects of the ABCG2 c.421 C > A SNP on the pharmacokinetics of simvastatin were reported by Keskitalo Jenni E et al. [41]. An association with simvastatin lactone pharmacokinetics was observed, but not with simvastatin acid. Alleles have been complemented to the plus chromosomal strand. Genotype TT is associated with increased exposure (area under the curve, AUC, reflecting the actual body exposure to drug after administration [42]) to drug when treated with simvastatin in healthy individuals as compared to genotypes GG + GT. Participants were selected 
that did not carry the CYP2C9* 3 allele or CYP3A5 g.6986 A allele, and did not have the SLCO1B1 521CC genotype. Genotyping for ABCG2 polymorphisms could help in predicting simvastatin pharmacokinetics and its dose for an individual patient. However, adverse drug reaction was not investigated in that study, and if this biomarker could predict an increased risk of simvastatin-induced myopathy is still unclear.

Adverse drug reactions occur almost daily in hospitals and can adversely affect a patient's quality of life [43]. Focusing on the association between genetic polymorphisms and simvastatin-related myopathy, a narrative synthesis of evidence was conducted. The current evidence does not allow to perform a meta-analysis, although this would have been the best approach to investigate the study question. The single SNP, rs4149056T > C, within SLCO1B1 increases the risk of muscle toxicity, and the evidence of this association is of high quality. The association has been reproduced in randomized trials and clinical practice-based cohorts. Modest increases in myopathy risk exist in patients with the $\mathrm{C}$ allele at SLC01B1 rs4149056, even at lower simvastatin doses (40 mg daily). Therefore, alternate statin drugs should be considered if optimal efficacy is not achieved with a lower dose of simvastatin. We believe that patients should be prospectively genotyped for rs4149056 prior to simvastatin therapy or an alternative solution (e.g. pravastatin or rosuvastatin) prescribed. As for other candidate genetic markers, such as ABCB1, GATM, HTB3B, HTR7, R2R, HLA$B 1{ }^{*} 04: 06$, additional studies will be needed to confirm this association.

\section{Authors' Contribution}

$X$. Xu and Z. Zhao conceived and designed this systematic review. X. Li, S. Sun and Z. Zhao selected the studies. X. Li and W. Li extracted the data and assessed the quality. X. Li and S. Sun wrote the manuscript. S. Sun and Z. Zhao supervised the quality of the study. All authors read and approved the final manuscript.

\section{Acknowledgements}

This project was supported by a grant from the National Natural Science Foundation of China (81503157), the Beijing Municipal Administration of Hospitals' Youth Programme (QML20170506), and the Beijing Municipal Administration of Hospitals (ZYLX201808).

\section{Conflict of Interest}

All authors declare no conflict of interest.

\section{References}

[1] Taylor F, Huffman MD, Macedo AF et al. Statins for the primary prevention of cardiovascular disease. Cochrane Database Syst Rev 2013; CD004816

[2] Naci H, Brugts ], Ades T. Comparative tolerability and harms of individual statins: A study-level network meta-analysis of 246955 participants from 135 randomized, controlled trials. Circ Cardiovasc Qual Outcomes 2013; 6: 390-399
[3] Abd TT, Jacobson TA. Statin-induced myopathy: A review and update. Expert Opin Drug Saf 2011; 10: 373-387

[4] Pasanen MK, Neuvonen M, Neuvonen PJ et al. SLCO1B1 polymorphism markedly affects the pharmacokinetics of simvastatin acid. Pharmacogenet Genomics 2006; 16: 873-879

[5] Group SC, Link E, Parish S et al. SLCO1B1 variants and statin-induced myopathy--A genomewide study. N Engl J Med 2008; 359: 789-799

[6] Ramsey LB, Johnson SG, Caudle KE et al. The clinical pharmacogenetics implementation consortium guideline for SLCO1B1 and simvastatininduced myopathy: 2014 update. Clin Pharmacol Ther 2014; 96: 423-428

[7] Voora D, Shah SH, Spasojevic I et al. The SLCO1B1 * 5 genetic variant is associated with statin-induced side effects. J Am Coll Cardiol 2009; 54 : 1609-1616

[8] Brunham LR, Lansberg PJ, Zhang L et al. Differential effect of the rs4149056 variant in SLCO1B1 on myopathy associated with simvastatin and atorvastatin. Pharmacogenomics J 2012; 12: 233-237

[9] Tsamandouras N, Dickinson G, Guo Y et al. Development and application of a mechanistic pharmacokinetic model for simvastatin and its active metabolite simvastatin acid using an integrated population PBPK approach. Pharm Res 2015; 32: 1864-1883

[10] Fiegenbaum M, da Silveira FR, Van der Sand CR et al. The role of common variants of ABCB1, CYP3A4, and CYP3A5 genes in lipidlowering efficacy and safety of simvastatin treatment. Clin Pharmacol Ther 2005; 78: 551-558

[11] Becker ML, Visser LE, van Schaik RH et al. Influence of genetic variation in CYP3A4 and ABCB1 on dose decrease or switching during simvastatin and atorvastatin therapy. Pharmacoepidemiol Drug Saf 2010; 19: 75-81

[12] Mangravite LM, Engelhardt BE, Medina MW et al. A statin-dependent QTL for GATM expression is associated with statin-induced myopathy. Nature 2013; 502: 377-380

[13] Luzum JA, Kitzmiller JP, Isackson PJ et al. GATM polymorphism associated with the risk for statin-induced myopathy does not replicate in case-control analysis of 715 dyslipidemic individuals. Cell Metab 2015; 21: 622-627

[14] Ruano G, Thompson PD, Windemuth A et al. Physiogenomic association of statin-related myalgia to serotonin receptors. Muscle Nerve 2007; 36: 329-335

[15] Marciante KD, Durda JP, Heckbert SR et al. Cerivastatin, genetic variants, and the risk of rhabdomyolysis. Pharmacogenet Genomics 2011; 21: 280-288

[16] Hubacek JA, Adamkova V, Hruba P et al. Association between polymorphism within the RYR2 receptor and development of statin-associated myalgia/myopathy in the Czech population. Eur J Intern Med 2015; 26: 367-368

[17] Sai K, Kajinami K, Akao $\mathrm{H}$ et al. A possible role for HLA-DRB1 * 04:06 in statin-related myopathy in Japanese patients. Drug Metab Pharmacokinet 2016; 31: 467-470

[18] Stang A. Critical evaluation of the Newcastle-Ottawa scale for the assessment of the quality of nonrandomized studies in meta-analyses. Eur J Epidemiol 2010; 25: 603-605

[19] Abe T, Kakyo M, Tokui T et al. Identification of a novel gene family encoding human liver-specific organic anion transporter LST-1. J Biol Chem 1999; 274: 17159-17163

[20] Takeda M, Noshiro R, Onozato ML et al. Evidence for a role of human organic anion transporters in the muscular side effects of HMG-CoA reductase inhibitors. Eur J Pharmacol 2004; 483: 133-138

[21] Jones PM, George AM. The ABC transporter structure and mechanism: perspectives on recent research. Cell Mol Life Sci 2004; 61: 682-699

[22] Joncquel-Chevalier Curt M, Voicu PM, Fontaine M et al. Creatine biosynthesis and transport in health and disease. Biochimie 2015; 119: 146-165 
[23] Mareedu RK, Modhia FM, Kanin El et al. Use of an electronic medical record to characterize cases of intermediate statin-induced muscle toxicity. Prev Cardiol 2009; 12: 88-94

[24] Xiao J, Tian X, Jones PP et al. Removal of FKBP12.6 does not alter the conductance and activation of the cardiac ryanodine receptor or the susceptibility to stress-induced ventricular arrhythmias. J Biol Chem 2007; 282: 34828-34838

[25] Davies PA, Pistis M, Hanna MC et al. The 5-HT3B subunit is a major determinant of serotonin-receptor function. Nature 1999; 397 : 359-363

[26] Hoyer D, Hannon JP, Martin GR. Molecular, pharmacological and functional diversity of 5-HT receptors. Pharmacol Biochem Behav 2002; 71: 533-554

[27] Ranasinghe S, Cutler S, Davis I et al. Association of HLA-DRB1-restricted CD4(+) T cell responses with HIV immune control. Nat Med 2013; 19: $930-933$

[28] Tirona RG, Leake BF, Merino G et al. Polymorphisms in OATP-C: Identification of multiple allelic variants associated with altered transport activity among European- and African-Americans. J Biol Chem 2001; 276: 35669-35675

[29] Kameyama Y, Yamashita K, Kobayashi K et al. Functional characterization of SLCO1B1 (OATP-C) variants, SLCO1B1 ${ }^{*} 5$, SLCO1B1 ${ }^{*} 15$ and SLCO1B1 * 15 + C1007G, by using transient expression systems of HeLa and HEK293 cells. Pharmacogenet Genomics 2005; 15: 513-522

[30] Pasanen MK, Fredrikson H, Neuvonen P] et al. Different effects of SLCO1B1 polymorphism on the pharmacokinetics of atorvastatin and rosuvastatin. Clin Pharmacol Ther 2007; 82: 726-733

[31] Thompson JF, Man M, Johnson KJ et al. An association study of 43 SNPs in 16 candidate genes with atorvastatin response. Pharmacogenomics J 2005; 5: 352-358

[32] Mwinyi ], Johne A, Bauer $S$ et al. Evidence for inverse effects of OATP-C (SLC21A6) 5 and 1b haplotypes on pravastatin kinetics. Clin Pharmacol Ther 2004; 75: 415-421

[33] Rodrigues AC, Perin PM, Purim SG et al. Pharmacogenetics of OATP transporters reveals that SLCO1B1 c.388A > G variant is determinant of increased atorvastatin response. Int J Mol Sci 2011; 12: 5815-5827
[34] Fu Q, Li YP, Gao Y et al. Lack of association between SLCO1B1 polymorphism and the lipid-lowering effects of atorvastatin and simvastatin in Chinese individuals. Eur J Clin Pharmacol 2013; 69: 1269-1274

[35] Prado Y, Saavedra N, Zambrano T et al. SLCO1B1 c.388A>G Polymorphism Is Associated with HDL-C Levels in Response to Atorvastatin in Chilean Individuals. Int J Mol Sci 2015; 16: 20609-20619

[36] Kadam P, Ashavaid TF, Ponde CK et al. Genetic determinants of lipid-lowering response to atorvastatin therapy in an Indian population. J Clin Pharm Ther 2016; 41: 329-333

[37] Wen JXiong Y. OATP1B1 388A>G polymorphism and pharmacokinetics of pitavastatin in Chinese healthy volunteers. J Clin Pharm Ther 2010; 35: 99-104

[38] Wang D, Johnson AD, Papp AC et al. Multidrug resistance polypeptide 1 (MDR1, ABCB1) variant 3435C > T affects mRNA stability. Pharmacogenet Genomics 2005; 15: 693-704

[39] Leschziner GD, Andrew T, Pirmohamed M et al. ABCB1 genotype and PGP expression, function and therapeutic drug response: $A$ critical review and recommendations for future research. Pharmacogenomics ] 2007; 7: 154-179

[40] Kajinami K, Brousseau ME, Ordovas JM et al. Polymorphisms in the multidrug resistance-1 (MDR1) gene influence the response to atorvastatin treatment in a gender-specific manner. Am J Cardiol 2004; 93: $1046-1050$

[41] Keskitalo JE, Pasanen MK, Neuvonen PJ et al. Different effects of the ABCG2 c.421C > A SNP on the pharmacokinetics of fluvastatin, pravastatin and simvastatin. Pharmacogenomics 2009; 10: 1617-1624

[42] Srinivas NR. Limited sampling strategy for the prediction of area under the curve (AUC) of statins: Reliability of a single time point for auc prediction for pravastatin and simvastatin. Drug Res (Stuttg) 2016; 66: 82-93

[43] Alaei M, Najmi AK, Kausar $\mathrm{H}$ et al. A prospective research study of anti-glaucoma drugs prescribing, utilization pattern and adverse drug reaction recording in a university hospital. Drug Res (Stuttg) 2015; 65: $164-168$ 